\title{
Simulation of photoelectron spectra with anharmonicity fully included: Application to the $\tilde{X}^{2} A_{2} \leftarrow \tilde{X}^{1} A_{1}$ band of furan
}

\author{
Sean Bonness and Bernard Kirtman \\ Department of Chemistry and Biochemistry, University of California, Santa Barbara, California 93106 \\ Miquel Huix, Angel J. Sanchez, and Josep M. Luis ${ }^{\text {a) }}$ \\ Institute of Computational Chemistry, University of Girona, Campus de Montilivi, 17071 Girona, Catalonia, \\ Spain and Department of Chemistry, University of Girona, Campus de Montilivi, 17071 Girona, \\ Catalonia, Spain
}

(Received 6 April 2006; accepted 11 May 2006; published online 5 July 2006)

Using a new unconventional procedure for calculating Franck-Condon factors with anharmonicity fully included the $\widetilde{X}^{2} A_{2} \leftarrow \widetilde{X}^{1} A_{1}$ band in the photoelectron spectrum of furan (and deuterated furan) was simulated at the second-order perturbation theory level. All 21 vibrational modes were considered but, in the end, only 4 are required to accurately reproduce the spectrum. Except for our own recent work on ethylene such calculations have been previously limited to tri- or tetraatomic molecules. Most of the effect of anharmonicity is accounted for in first order, although second-order corrections to the vibrational frequencies are important. Based on these simulations we were able to improve upon and extend previous assignments as well as suggest further measurements. (C) 2006 American Institute of Physics. [DOI: 10.1063/1.2210479]

\section{INTRODUCTION}

The assignment and interpretation of vibronic transitions in UV absorption, photoelectron, and circular dichroism spectra have become of increasing importance with the advent of sophisticated high resolution measurement techniques. Accurate $a b$ initio simulations can play a key role in this task. The transition intensities, in particular, are governed in first approximation by Franck-Condon factors (FCFs). ${ }^{1,2}$ Although there are other smaller contributions due to Herzberg-Teller factors, ${ }^{3}$ and possible non-BornOppenheimer effects, ${ }^{4}$ the FCFs are typically dominant and we focus on their determination in this paper. Apart from the spectral applications mentioned above it should be noted that the same integrals govern numerous other processes, such as radiationless transitions and electron transfer.

For a long time the calculation of FCFs was restricted to the harmonic oscillator level of approximation. ${ }^{5-7}$ In fact, important advances at that level continue to be made as, for example, in the treatment of Duschinsky rotations. ${ }^{8}$ Recently, however, increased attention has been devoted towards taking into account vibrational anharmonicity. On the one hand, new formulations have been developed ${ }^{9-16}$ as summarized previously ${ }^{17}$ while, on the other, efficient computational algorithms/codes have been implemented. ${ }^{18}$ Considerable progress has been made, particularly for small molecules. However, it still remains difficult to incorporate anharmonic coupling between different modes for molecules containing more than just a few atoms, i.e., a maximum of six vibrational degrees of freedom. ${ }^{18,19}$

A short time ago we formulated and implemented ${ }^{17,20}$ a

\footnotetext{
${ }^{a)}$ Author to whom correspondence should be addressed. Electronic mail: josepm.luis@udg.es
}

nonconventional procedure that makes it possible, for the first time, to calculate FCFs of larger polyatomic molecules including mode-mode anharmonic coupling as well as Duschinsky rotations. Our method has been successfully applied to the photoelectron spectra of $\mathrm{ClO}_{2}$ and ethylene. Here we study the photoelectron spectrum of furan, a molecule with almost twice as many vibrational degrees of freedom as ethylene. Furan is of interest not only because of its relatively large size, as far as FCF calculations that fully include anharmonicity are concerned, but also because of the quality and quantity of experimental data as well as the opportunity to improve upon earlier assignments. Furan currently plays an important role in organic chemistry and molecular biology, as well as in materials science where it is a building block for promising novel materials. Both the normal and the deuterated species are considered here.

A brief review of the theoretical treatment is provided in Sec. II along with the computational details for furan. Then in Sec. III our results are analyzed in detail. Finally, we present the key conclusions in Sec. IV and indicate the direction of further developments.

\section{THEORY AND COMPUTATIONAL DETAILS}

In our procedure the Franck-Condon integrals are obtained by solving a set of homogeneous linear simultaneous equations $^{17,20}$

$$
\sum_{\mu_{e}}^{M} S_{\nu_{g} \mu_{e}}\left[\left\langle\psi_{\mu_{e}}^{e}\left|\hat{V}^{g}-\hat{V}^{e}\right| \psi_{\nu_{e}}^{e}\right\rangle+\left(E_{\nu_{e}}^{e}-E_{\nu_{g}}^{g}\right) \delta_{\mu_{e} \nu_{e}}\right]=0, \quad \forall \nu_{g},
$$

together with the normalization condition $\left(\mathbf{S}_{\mathbf{v} s}^{\dagger} \mathbf{S}_{\mathbf{v}^{g}}=1\right)$. Here $g$ refers to the ground electronic state and $e$ to the excited 
electronic state. For a given $\nu_{g}, \mathbf{S}_{\boldsymbol{\nu}^{g}}$ is the eigenvector containing the set of Franck-Condon overlap integrals

$$
S_{\nu_{e} \nu_{g}}=S_{\nu_{g} \nu_{e}}=\left\langle\psi_{\nu_{e}}^{e} \mid \psi_{\nu_{g}}^{g}\right\rangle=\left\langle\psi_{\nu_{g}}^{g} \mid \psi_{\nu_{e}}^{e}\right\rangle
$$

for all $\nu_{e}$. In Eq. (1) $\hat{V}^{g}$ and $\hat{V}^{e}$ are the ground and excited state vibrational potential energy functions, $\left|\psi_{v_{e}}^{e}\right\rangle$ is a vibronic wave function, $E_{\nu_{g}}^{g}$ and $E_{\nu_{e}}^{e}$ are vibronic energies, and $\delta_{\mu_{e} \nu_{e}}$ is the Kronecker delta. In practice, of course, the set of all $\nu_{e}$ must be truncated to a finite dimension $M$. Finally, the FCFs are given by the square of the Franck-Condon integrals, i.e., $F_{\nu_{g} \mu_{e}}=S_{\nu_{g} \mu_{e}}^{2}$.

The normal coordinates of the electronic excited state $\left(\mathbf{Q}^{\mathbf{e}}\right)$ are different from the normal coordinates of the ground state $\left(\mathbf{Q}^{\mathbf{g}}\right)$. One may express the transformation from $\mathbf{Q}^{\mathbf{e}}$ to $\mathbf{Q}^{\mathbf{g}}$ as the sum of a term due to the change in the equilibrium geometry (K) plus a term arising from the generalized (Duschinsky) rotation of the coordinates, i.e.,

$$
\mathbf{Q}^{\mathbf{g}}=\mathbf{K}+\mathbf{J} \mathbf{Q}^{\mathbf{e}} .
$$

Using the latter relation it is straightforward to write the normal coordinate expansion of the potential energy difference in Eq. (1) as a function solely of the excited electronic state normal coordinates. ${ }^{17,20}$

The potential energy integral in Eq. (1) is easily evaluated if $\hat{V}^{g}\left(\mathbf{Q}^{\mathbf{g}}\right)$ and $\hat{V}^{e}\left(\mathbf{Q}^{\mathbf{e}}\right)$ are both approximated by a harmonic normal coordinate expansion. However, when anharmonicity must be taken into account the situation becomes more complicated. The least computationally demanding method of taking into account anharmonicity is by means of vibrational perturbation theory based on the harmonic oscillator zeroth-order model. Another alternative is the vibrational self-consistent field (VSCF) treatment. ${ }^{21-24}$ But the VSCF treatment includes mode-mode anharmonic coupling only in the mean field approximation. The simplest method for going beyond VSCF, i.e., for introducing vibrational correlation, is second-order Møller-Plesset perturbation theory (VMP2). ${ }^{25,26}$ Other methods such as vibrational configuration interaction ${ }^{27-31}$ or vibrational coupled cluster theory ${ }^{32}$ are much more computationally intensive. For the molecule considered here, namely, furan, the perturbation approach is far easier to carry out, assuming rapid convergence, and that is the method we will employ.

If the harmonic model is taken as the zeroth-order approximation, then the first-order perturbation equation derived from Eq. (1) is given by ${ }^{17,20}$

$$
\begin{aligned}
\sum_{\mu_{e}} S_{\nu_{g} \mu_{e}}^{(1)}\left[\left\langle\psi_{\mu_{e}}^{e}\left|\hat{V}^{g}-\hat{V}^{e}\right| \psi_{\nu_{e}}^{e}\right\rangle+\left(E_{\nu_{e}}^{e}-E_{\nu_{g}}^{g}\right) \delta_{\mu_{e} \nu_{e}}\right]^{(0)} \\
\quad+\sum_{\mu_{e}} S_{\nu_{g} \mu_{e}}^{(0)}\left[\left\langle\psi_{\mu_{e}}^{e}\left|\hat{V}^{g}-\hat{V}^{e}\right| \psi_{\nu_{e}}^{e}\right\rangle+\left(E_{\nu_{e}}^{e}-E_{\nu_{g}}^{g}\right) \delta_{\mu_{e} \nu_{e}}\right]^{(1)}=0,
\end{aligned}
$$

where

$$
\begin{aligned}
\left\langle\psi_{\mu_{e}}^{e}\left|\hat{V}^{g}-\hat{V}^{e}\right| \psi_{\nu_{e}}^{e}\right\rangle^{(1)} & \\
= & \left\langle\psi_{\mu_{e}}^{e(1)}\left|\left(\hat{V}^{g}-\hat{V}^{e}\right)^{(0)}\right| \psi_{\nu_{e}}^{e(0)}\right\rangle+\left\langle\psi_{\mu_{e}}^{e(0)}\left|\left(\hat{V}^{g}-\hat{V}^{e}\right)^{(1)}\right| \psi_{\nu_{e}}^{e(0)}\right\rangle \\
& +\left\langle\psi_{\mu_{e}}^{e(0)}\left|\left(\hat{V}^{g}-\hat{V}^{e}\right)^{(0)}\right| \psi_{\nu_{e}}^{e(1)}\right\rangle .
\end{aligned}
$$

Since we define the cubic and quartic terms of $\hat{V}^{g}$ and $\hat{V}^{e}$ as first- and second-order perturbations, respectively, the firstorder corrections to $E_{\nu_{e}}^{e}$ and $E_{\nu_{g}}^{g}$ vanish. This choice is not unique; other partitions could be used. Quintic and higherorder terms are not considered.

The first-order wave function $\psi_{\mu_{e}}^{e(1)}$, that appears in Eq. (4), is calculated using the standard perturbation theory expression. ${ }^{17,20}$ Then, after imposing the first-order normalization condition $\left(\mathbf{S}_{\mathbf{v}^{g}}^{(1)} \mathbf{S}_{\mathbf{v}^{g}}^{(0)}=0\right)$, Eq. (4) reduces to a set of $M-1$ inhomogeneous linear equations which can be easily solved. The resulting first-order corrections to the FCFs are just twice the product of the zeroth-order and first-order Franck-Condon integrals $\left(F_{\nu_{g} \mu_{e}}^{(1)}=2 S_{\nu_{g} \mu_{e}}^{(0)} S_{\nu_{g} \mu_{e}}^{(1)}\right)$. This procedure is readily extended to second- (and higher-) order corrections. $^{20}$

The most critical step in our method is the truncation of the vibrational basis set for the excited electronic state. For accurate and efficient computation we want to include all vibrational basis functions that give rise to significant contributions to the Franck-Condon intensity, but no others. Our algorithm for doing so involves an iterative buildup which increases the range of vibrational quantum numbers while, simultaneously, removing unnecessary functions. ${ }^{17,20}$ For each trial basis, the associated set of FCFs is determined by solving the resulting linear simultaneous equations to the desired order of perturbation theory. All vibrational states corresponding to FCFs smaller than a certain threshold are, then, marked for exclusion in further basis set augmentations. The iterative procedure converges when the set of nonnegligible FCFs is the same in two consecutive iterations. By excluding vibrational wave functions in this manner we drastically reduce the growth of the basis set thereby leading to a major improvement in efficiency. Such an algorithm is vital for calculating FCFs of a molecule such as furan which contains 21 normal modes that can be coupled by mechanical anharmonicity. Indeed, as far as we know, until now there have been no FCF calculations that include the effect of anharmonic mode-mode coupling for molecules containing more than four atoms except for our own previous simulation of the $\tilde{X}^{2} B_{3 u} \leftarrow \tilde{X}^{1} A_{g}$ band in the photoelectron spectrum of ethylene.

Ab initio electronic structure calculations for the ground states of neutral and cationic furan were performed at the (U)B3LYP 6-311++G( $d, p)$ (Refs. 33-36) level. Cubic and quartic vibrational force constants were determined by numerical differentiation of quadratic force constants obtained from the GAUSSIAN 03 suite of programs. ${ }^{37}$ Vibrational displacements of \pm 0.04 and \pm 0.08 a.u. were employed for this purpose together with the Romberg method ${ }^{38}$ to reduce numerical error. Theoretical FCFs and vibrational energies were used to determine the relative peak intensities and positions in the He I photoelectron (PE) spectrum except that 
TABLE I. Equilibrium geometrical parameters for neutral and cationic furan. All quantities are in angstroms and degrees (see Fig. 1).

\begin{tabular}{|c|c|c|c|c|c|c|c|c|}
\hline & \multicolumn{6}{|c|}{$\mathrm{C}_{4} \mathrm{H}_{4} \mathrm{O} \tilde{X}^{1} A_{1}\left(C_{2 v}\right)$} & \multicolumn{2}{|c|}{$\mathrm{C}_{4} \mathrm{H}_{4} \mathrm{O}^{+} \tilde{X}^{2} A_{2}\left(C_{2 v}\right)$} \\
\hline & $\mathrm{BL} 3 \mathrm{YP} / 6-311++\mathrm{G}(d, p)$ & $\begin{array}{c}\text { B3LYP/ } \\
6-311+\mathrm{G}(3 d f)^{\mathrm{a}}\end{array}$ & $\mathrm{B} 97-1 / \mathrm{TZ} 2 \mathrm{P}+{ }^{\mathrm{b}}$ & $\mathrm{MP} 2 / \mathrm{cc}-\mathrm{pVTZ} \mathrm{C}^{\mathrm{c}}$ & $\begin{array}{c}\text { CCSD/ } \\
\text { cc-pVDS+ }{ }^{\mathrm{d}}\end{array}$ & Expt. $^{\mathrm{e}}$ & $\begin{array}{c}\text { B3LYP/ } \\
6-311++\mathrm{G}(d, p)\end{array}$ & $\begin{array}{c}\text { B3LYP/ } \\
6-311+\mathrm{G}(3 d)^{\mathrm{a}}\end{array}$ \\
\hline$r(1 \mathrm{O}-2 \mathrm{C})$ & 1.363 & 1.360 & 1.361 & 1.359 & 1.371 & 1.362 & 1.347 & 1.345 \\
\hline$r(2 \mathrm{C}-3 \mathrm{C})$ & 1.358 & 1.354 & 1.358 & 1.364 & 1.369 & 1.361 & 1.414 & 1.410 \\
\hline$r(3 \mathrm{C}-4 \mathrm{C})$ & 1.435 & 1.432 & 1.435 & 1.426 & 1.451 & 1.431 & 1.381 & 1.377 \\
\hline$r(2 \mathrm{C}-6 \mathrm{H})$ & 1.077 & 1.077 & 1.077 & 1.074 & 1.087 & 1.075 & 1.082 & 1.081 \\
\hline$r(3 \mathrm{C}-7 \mathrm{H})$ & 1.078 & 1.078 & 1.078 & 1.075 & 1.089 & 1.077 & 1.080 & 1.079 \\
\hline$\angle(5 \mathrm{C}-1 \mathrm{O}-2 \mathrm{C})$ & 106.9 & 106.9 & 106.8 & 106.7 & 106.6 & 106.7 & 106.7 & 106.7 \\
\hline$\angle(1 \mathrm{O}-2 \mathrm{C}-3 \mathrm{C})$ & 110.4 & 110.4 & 110.6 & 110.6 & 110.8 & 110.7 & 110.6 & 110.5 \\
\hline$\angle(2 \mathrm{C}-3 \mathrm{C}-4 \mathrm{C})$ & 106.1 & 106.1 & 106.0 & 106.1 & 105.9 & 106.0 & 106.1 & 106.1 \\
\hline$\angle(1 \mathrm{O}-2 \mathrm{C}-6 \mathrm{H})$ & 115.9 & 115.9 & 116.0 & 115.9 & 115.8 & 115.9 & 116.6 & 116.6 \\
\hline$\angle(2 \mathrm{C}-3 \mathrm{C}-7 \mathrm{H})$ & 126.5 & 126.5 & 126.4 & 126.1 & 126.3 & 126.1 & 125.4 & 125.4 \\
\hline
\end{tabular}

${ }^{\mathrm{a}}$ Reference 45 .

${ }^{\mathrm{b}}$ Reference 46.

${ }^{\mathrm{c}}$ Reference 47.

the first peak was shifted to the experimental value, which occurs at the adiabatic ionization energy (AIE) of $8.883 \mathrm{eV}$ measured by Derrick et al. ${ }^{39}$ In order to simulate the vibronic bands we utilized Gaussian functions with a full width at half maximum of $15 \mathrm{meV}$.

The shape of a photoelectron band suffers large changes when the geometry of the neutral or cation is lightly perturbed. In order to avoid errors from this source in our simulations we utilized the experimental geometry of neutral furan $^{40}$ together with the geometry from an iterative FranckCondon analysis ${ }^{7}$ (IFCA) for the cation. The IFCA procedure optimizes geometrical parameters by obtaining a best fit between the simulated and the experimental spectrum. Calculations performed by Chau and co-workers ${ }^{41-44}$ have shown that, for the purpose of comparing simulated with observed spectra, it is best to use the IFCA geometry of the excited state. It is justified to use this geometry only when there is good agreement with the geometry from high level $a b$ initio calculations which is the case in this paper. The experimental values for the IFCA optimizations were taken from measurements of Derrick et al. ${ }^{39}$

\section{RESULTS}

Both neutral and cationic furan have a planar $C_{2 \nu}$ equilibrium geometry. The symmetry of the ground electronic state of the neutral is ${ }^{1} A_{1}$. Ionization from the $1 a_{2} \pi$ orbital, which is the highest occupied molecular orbital (HOMO), leads to the $\tilde{X}^{2} A_{2}$ state of the cation. Table I contains our optimized (U)B3LYP/6-311++ $\mathrm{G}(d, p)$ equilibrium geometry parameters for both species, as well as the values obtained in other theoretical studies. The latter includes the B3LYP/6-311+G(3df) calculations of Yan et al. ${ }^{45}$ the density functional theory (DFT) calculations of Burcl et al. ${ }^{46}$ obtained with the B97-1 functional and the augmented triple $\zeta$ basis set $(\mathrm{TZ2P}+)$ : the MP2/cc-pVTZ values of Mellouki et al.; ${ }^{47}$ and the CCSD/cc-pVDZ+ calculations of Gromov et $a{ }^{48}$ Table I also contains experimental values of Nygaard $e t$ $a l .^{49}$ for the geometrical parameters of neutral furan, which are in very good to excellent agreement with all the $a b$ initio

\section{${ }^{\mathrm{d}}$ Reference 48}

${ }^{\mathrm{e}}$ Reference 49 .

values. For the cation, our theoretical values are very similar to those of Yan et al. As expected, the DFT geometrical parameters of the cation show a considerable increase (decrease) of the $2 \mathrm{C}-3 \mathrm{C}(3 \mathrm{C}-4 \mathrm{C})$ bond lengths when the $a_{2} \pi$ electron of furan is removed (see Fig. 1).

In Table II we present our $a b$ initio harmonic frequencies for neutral and cationic furan molecule together with the $a b$ initio values of El-Azhary and Suter, ${ }^{50}$ Mellouki et al., ${ }^{47}$ and Gromov et al. $^{48}$ for the neutral molecule. Also shown are the experimental fundamental vibrational frequencies of Mellouki et $a l .^{47}$ for the neutral and three totally symmetric fundamental vibrational frequencies of the cation extracted from the photoelectron spectra measured by Derrick et al. The $a b$ initio harmonic frequencies are in good agreement with one another. They are also a satisfactory agreement with the observed fundamental frequencies being almost uniformly a bit higher.

In our preliminary calculations at the harmonic and firstorder perturbation theory (PT1) levels we considered all 21 modes in constructing the vibrational basis set. At this point our basis set truncation algorithm was essential. The results indicated that all non-negligible FCFs could be associated with just three $a_{1}$ modes, which correspond to our $a b$ initio harmonic frequencies of $1445 \mathrm{~cm}^{-1}\left(\omega_{4}\right), 1114 \mathrm{~cm}^{-1}\left(\omega_{6}\right)$, and $883 \mathrm{~cm}^{-1}\left(\omega_{8}\right)$. Figures 2(a)-2(c) depict the displace-

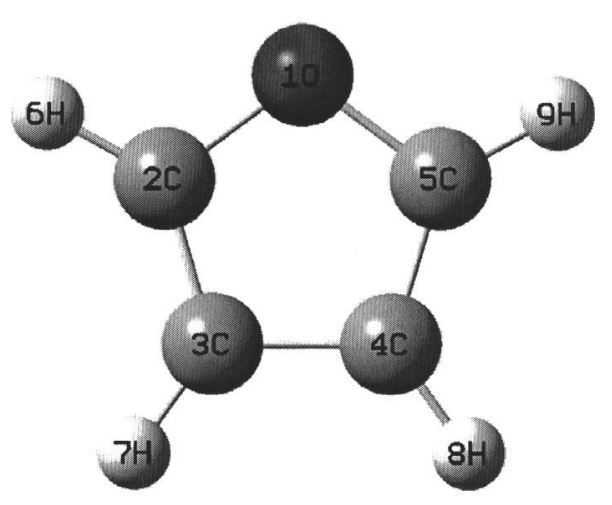

FIG. 1. Geometry of neutral and cation furan. 
TABLE II. Theoretical values of harmonic frequencies $\left(\mathrm{cm}^{-1}\right)$ and experimental values of fundamental vibrational frequencies $\left(\mathrm{cm}^{-1}\right)$ of neutral and cationic furan and deuterated furan.

\begin{tabular}{|c|c|c|c|c|c|c|c|c|c|c|}
\hline \multirow[b]{2}{*}{ Label } & \multirow[b]{2}{*}{ Sym. } & \multicolumn{5}{|c|}{$\mathrm{C}_{4} \mathrm{H}_{4} \mathrm{O} \tilde{X}^{1} A_{1}$} & \multicolumn{2}{|c|}{$\mathrm{C}_{4} \mathrm{H}_{4} \mathrm{O}^{+} \tilde{X}^{2} A_{2}$} & \multirow{2}{*}{\multicolumn{2}{|c|}{$\frac{\mathrm{C}_{4} \mathrm{D}_{4} \mathrm{O} \tilde{X}^{1} A_{1}}{\mathrm{~B} 3 \mathrm{LYP} /} \frac{\mathrm{C}_{4} \mathrm{D}_{4} \mathrm{O}^{+} \tilde{X}^{2} A_{2}}{6-311 \mathrm{G}++(d, p)}$}} \\
\hline & & $\begin{array}{c}\text { B3LYP/ } \\
6-311++\mathrm{G}(d, p)\end{array}$ & $\begin{array}{l}\text { B3LYP/ } \\
\text { cc-pVTZ }\end{array}$ & $\begin{array}{c}\text { MP2/ } \\
\text { cc-pVTZ }\end{array}$ & $\begin{array}{c}\mathrm{CCSD} / \\
\mathrm{cc-pVDZ}+{ }^{\mathrm{c}}\end{array}$ & Expt. $^{b}$ & $\begin{array}{c}\text { B3LYP/ } \\
6-311++\mathrm{G}(d, p)\end{array}$ & Expt. $^{\mathrm{a}}$ & & \\
\hline$\omega_{1}$ & $a_{1}$ & 3283 & 3279 & 3326 & 3312 & 3169 & 3262 & & 2453 & 2437 \\
\hline$\omega_{2}$ & $a_{1}$ & 3251 & 3251 & 3300 & 3283 & 3140 & 3248 & & 2405 & 2412 \\
\hline$\omega_{3}$ & $a_{1}$ & 1506 & 1513 & 1510 & 1546 & 1491 & 1511 & & 1438 & 1437 \\
\hline$\omega_{4}$ & $a_{1}$ & 1409 & 1415 & 1421 & 1427 & 1385 & 1445 & 1420 & 1310 & 1366 \\
\hline$\omega_{5}$ & $a_{1}$ & 1162 & 1167 & 1166 & 1165 & 1140 & 1164 & & 1076 & 1103 \\
\hline$\omega_{6}$ & $a_{1}$ & 1083 & 1086 & 1114 & 1082 & 1067 & 1114 & 1073 & 935 & 923 \\
\hline$\omega_{7}$ & $a_{1}$ & 1012 & 1015 & 1026 & 1011 & 995 & 1062 & & 801 & 817 \\
\hline$\omega_{8}$ & $a_{1}$ & 887 & 888 & 879 & 878 & 870 & 883 & 839 & 737 & 756 \\
\hline$\omega_{9}$ & $a_{2}$ & 880 & 893 & 868 & 874 & 864 & 963 & & 740 & 790 \\
\hline$\omega_{10}$ & $a_{2}$ & 730 & 739 & 726 & 739 & 722 & 837 & & 579 & 682 \\
\hline$\omega_{11}$ & $a_{2}$ & 609 & 618 & 613 & 599 & 600 & 476 & & 506 & 397 \\
\hline$\omega_{12}$ & $b_{1}$ & 850 & 858 & 839 & 855 & 838 & 898 & & 709 & 766 \\
\hline$\omega_{6}$ & $b_{1}$ & 755 & 762 & 761 & 761 & 745 & 766 & & 607 & 592 \\
\hline$\omega_{7}$ & $b_{1}$ & 618 & 625 & 632 & 606 & 603 & 497 & & 509 & 416 \\
\hline$\omega_{8}$ & $b_{2}$ & 3276 & 3272 & 3318 & 3304 & 3161 & 3251 & & 2438 & 2410 \\
\hline$\omega_{9}$ & $b_{2}$ & 3241 & 3241 & 3290 & 3271 & 3130 & 3240 & & 2391 & 2394 \\
\hline$\omega_{10}$ & $b_{2}$ & 1588 & 1595 & 1578 & 1671 & 1558 & 1402 & & 1518 & 1276 \\
\hline$\omega_{11}$ & $b_{2}$ & 1284 & 1255 & 1292 & 1289 & 1267 & 1294 & & 1150 & 1021 \\
\hline$\omega_{12}$ & $b_{2}$ & 1195 & 1199 & 1248 & 1216 & 1181 & 1035 & & 985 & 971 \\
\hline$\omega_{11}$ & $b_{2}$ & 1057 & 1062 & 1066 & 1062 & 1043 & 1005 & & 870 & 788 \\
\hline$\omega_{12}$ & $b_{2}$ & 893 & 894 & 884 & 884 & 873 & 788 & & 786 & 766 \\
\hline
\end{tabular}

${ }^{\mathrm{a}}$ Reference 50 .

${ }^{\mathrm{b}}$ Reference 47.

ments of the corresponding normal modes. While $\omega_{6}$ is an almost pure hydrogen atom bending mode, the $\omega_{4}$ and $\omega_{8}$ bends also involve important contributions from the heavy atoms. Our initial calculations agree with the experimental

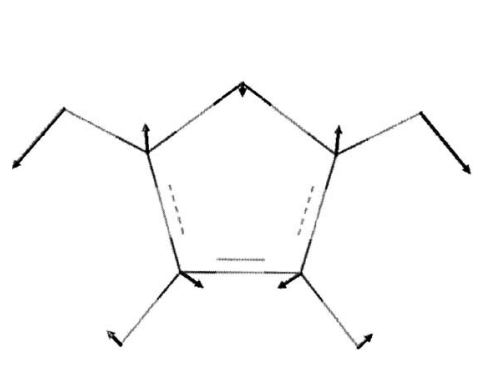

(A)

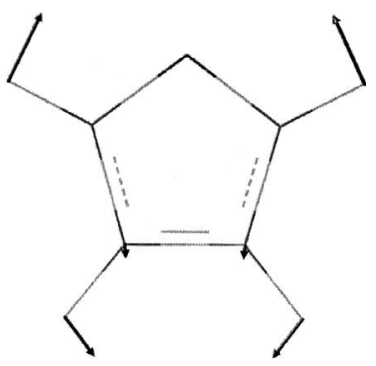

(B)
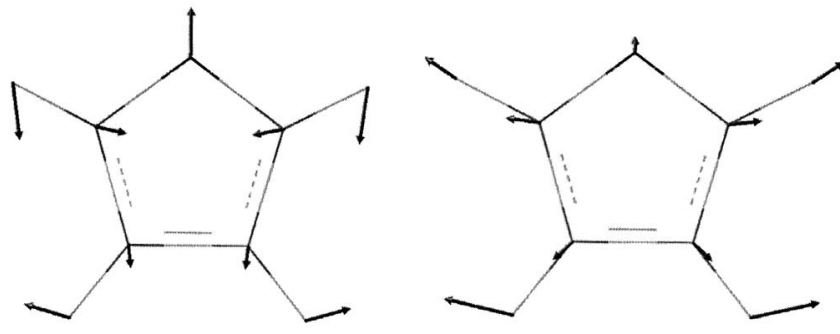

(C)

(D)

FIG. 2. Displacements associated with $a_{1} \omega_{4}(\mathrm{~A}), \omega_{6}(\mathrm{~B}), \omega_{8}(\mathrm{C})$, and $\omega_{5}(\mathrm{D})$ normal modes of $\mathrm{C}_{4} \mathrm{H}_{4} \mathrm{O}$.
${ }^{\mathrm{c}}$ Reference 48 .

${ }^{\mathrm{d}}$ Reference 39.

data obtained by Derrick et al. ${ }^{39}$ who observed progressions of these three $a_{1}$ modes in the first band of the photoelectron spectrum.

Although only three modes are significant at the harmonic and PT1 levels, at second-order perturbation theory (PT2) level, the $a_{1}$ mode with $a b$ initio harmonic frequency of $1164 \mathrm{~cm}^{-1}\left(\omega_{5}\right)$ [see Fig. 2(d)] is also significant. The FCFs associated with this mode are negligible, but the coupling with the other three active $a_{1}$ modes decreases the frequency of the high frequency peaks. The effect of this coupling can be seen in Figs. 3 and 4 which contain our $\tilde{X}^{2} A_{2}$ $\leftarrow \widetilde{X}^{1} A_{1}$ band simulated at the harmonic and PT2 levels using either three or four modes as the vibrational basis set.

In Table III we present the theoretical FCFs and frequencies evaluated at the PT2 level using four modes $\left(\omega_{4} \omega_{5} \omega_{6} \omega_{8}\right)$. Our assignment of the low energy peaks is given in Table IV and the high energy peaks in Table V. A comparison with Derrick et al. ${ }^{39}$ who labeled 8 of the 11 observed peaks, is provided in Table IV. In the experimental spectrum Derrick et al. ${ }^{39}$ identified three progressions. The one with the most intense peaks is $\left(\omega_{4} 000\right)=(0000),(1000)$, and (2000). They also identified the first two peaks of the $\left(\omega_{4} 010\right)$ progression [i.e., $(0010)$ and (1010)], the first two peaks of the $\left(\omega_{4} 001\right)$ progression, and, finally, the (0011) peak. It was also noted that the (1001) peak is overlapped by (0020). Figures 3 and 4 and the data of Table IV show that exactly the same peaks are obtained from our FCF calculations. Furthermore, there is very good agreement between our PT2 theoretical peak positions and the experimental values. 


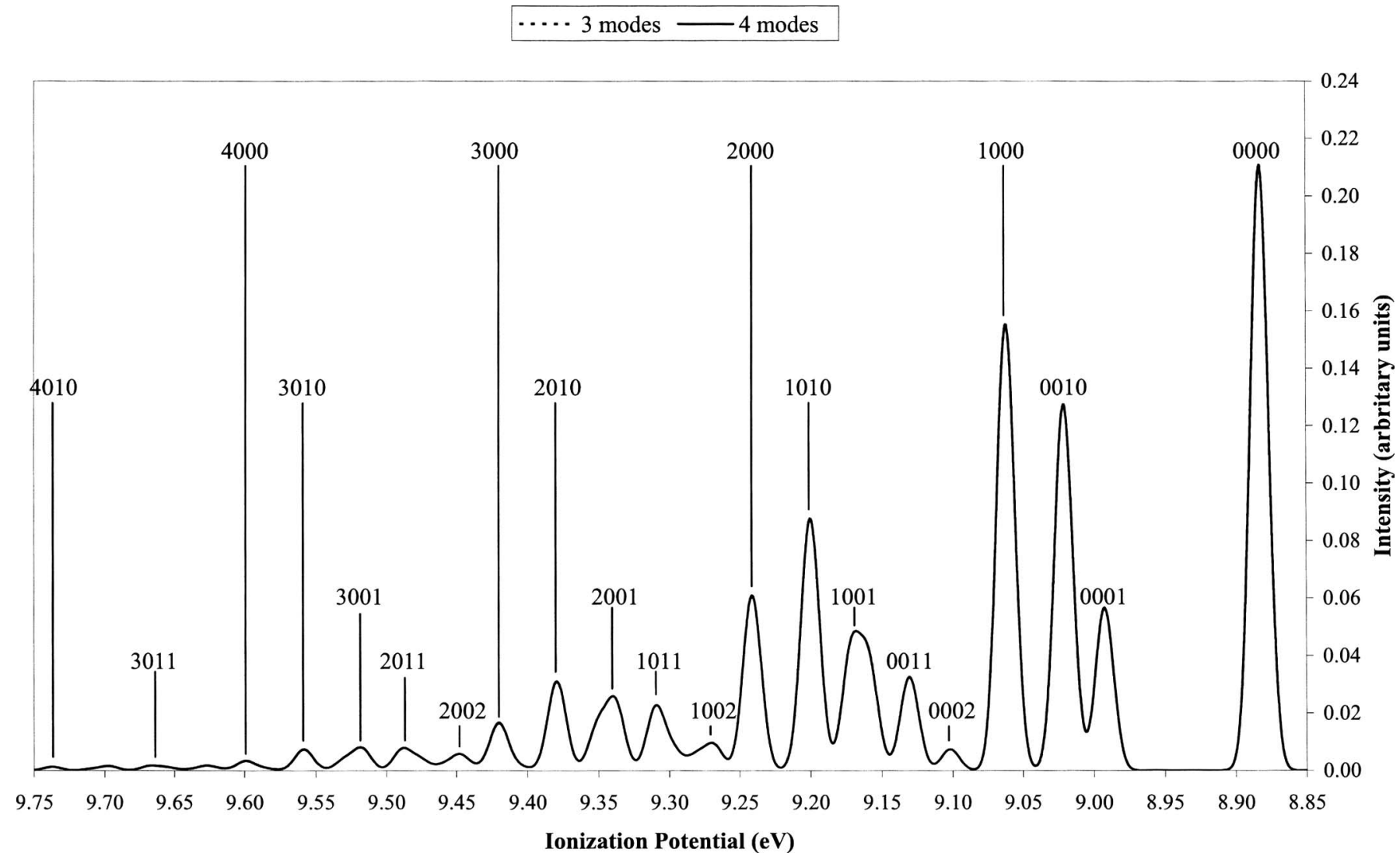

FIG. 3. (U)B3LYP/6-311++G $(d, p)$ harmonic simulation of the first band in the furan PE spectrum. The dashed and solid lines represent the spectra obtained with $\omega_{4} \omega_{6} \omega_{8}$ and $\omega_{4} \omega_{5} \omega_{6} \omega_{8}$ vibrational basis sets, respectively.

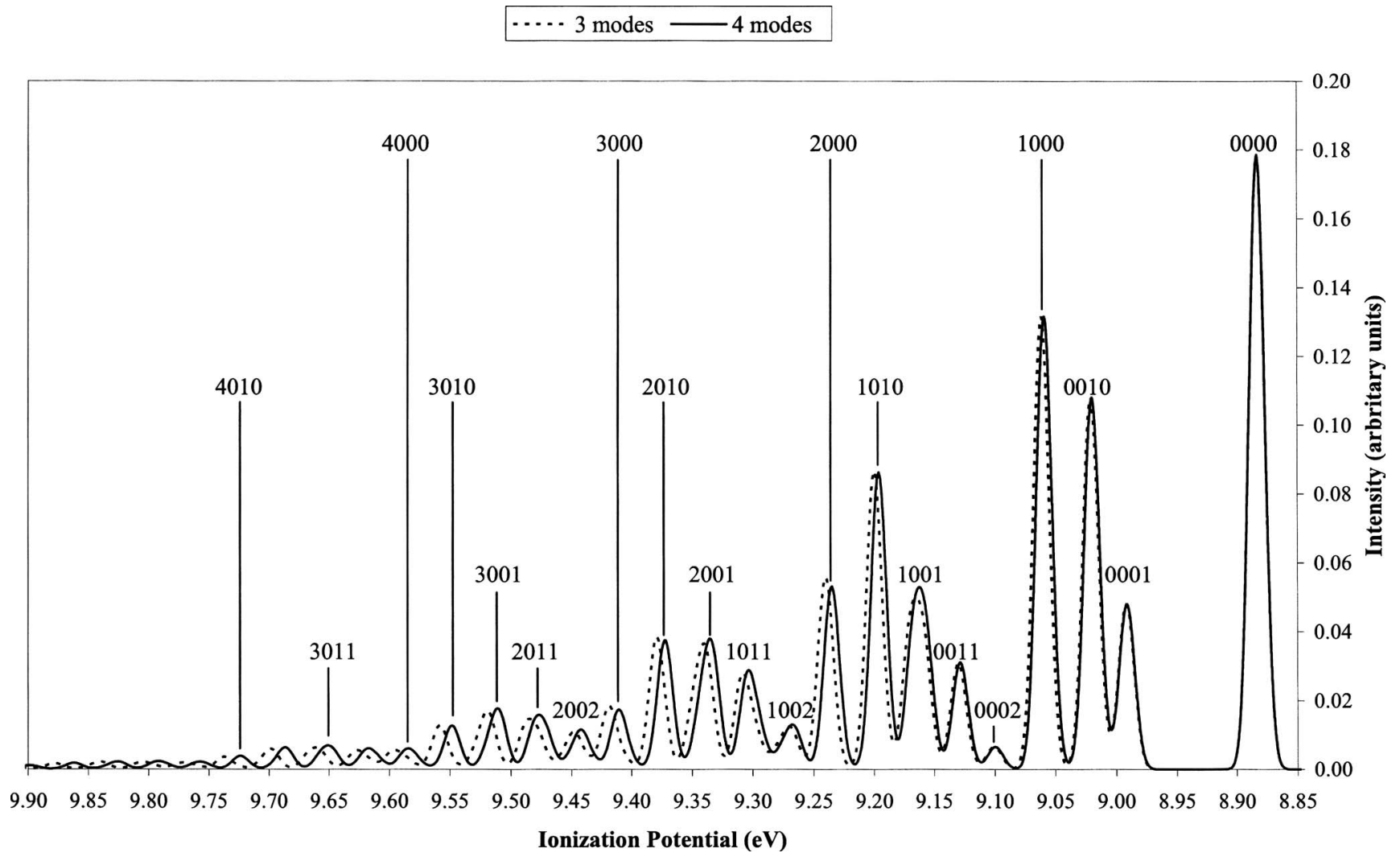

FIG. 4. (U)B3LYP/6-311++G $(d, p)$ PT2 simulation of the first band in the furan PE spectrum. The dashed and solid lines represent the spectra obtained with $\omega_{4} \omega_{6} \omega_{8}$ and $\omega_{4} \omega_{5} \omega_{6} \omega_{8}$ vibrational basis sets, respectively. 
TABLE III. (U)B3LYP/6-311++G( $d, p)$ frequencies and FCFs for the $\tilde{X}^{2} A_{2} \leftarrow \widetilde{X}^{1} A_{1}$ band of the furan PE spectrum computed at the PT2 level. The FCFs are in units so that the FCF vector is normalized. Only the FCFs, larger than $10^{-3}$ units are shown.

\begin{tabular}{|c|c|c|}
\hline$\omega_{4} \omega_{5} \omega_{6} \omega_{8}$ & Frequency $(\mathrm{eV})$ & FCF \\
\hline 0000 & 8.883 & $1.79 \times 10^{-1}$ \\
\hline 0001 & 8.991 & $4.81 \times 10^{-2}$ \\
\hline 0010 & 9.020 & $1.08 \times 10^{-1}$ \\
\hline 1000 & 9.059 & $1.32 \times 10^{-1}$ \\
\hline 0002 & 9.100 & $6.55 \times 10^{-3}$ \\
\hline 0011 & 9.128 & $3.12 \times 10^{-2}$ \\
\hline 0020 & 9.157 & $3.69 \times 10^{-2}$ \\
\hline 1001 & 9.167 & $3.71 \times 10^{-2}$ \\
\hline 1010 & 9.196 & $8.65 \times 10^{-2}$ \\
\hline 2000 & 9.235 & $4.90 \times 10^{-2}$ \\
\hline 0012 & 9.237 & $4.46 \times 10^{-3}$ \\
\hline 0021 & 9.266 & $1.11 \times 10^{-2}$ \\
\hline 1002 & 9.276 & $5.28 \times 10^{-3}$ \\
\hline 0030 & 9.294 & $9.23 \times 10^{-3}$ \\
\hline 1011 & 9.305 & $2.58 \times 10^{-2}$ \\
\hline 1020 & 9.333 & $3.21 \times 10^{-2}$ \\
\hline 2001 & 9.343 & $1.47 \times 10^{-2}$ \\
\hline 2010 & 9.372 & $3.57 \times 10^{-2}$ \\
\hline 0022 & 9.374 & $1.65 \times 10^{-3}$ \\
\hline 0031 & 9.403 & $2.90 \times 10^{-3}$ \\
\hline 3000 & 9.411 & $1.23 \times 10^{-2}$ \\
\hline 1012 & 9.413 & $3.86 \times 10^{-3}$ \\
\hline 0040 & 9.432 & $1.99 \times 10^{-3}$ \\
\hline 1021 & 9.442 & $9.99 \times 10^{-3}$ \\
\hline 2002 & 9.451 & $2.26 \times 10^{-3}$ \\
\hline 1030 & 9.471 & $9.09 \times 10^{-3}$ \\
\hline 2011 & 9.481 & $1.12 \times 10^{-2}$ \\
\hline 2020 & 9.510 & $1.52 \times 10^{-2}$ \\
\hline 3001 & 9.519 & $4.00 \times 10^{-3}$ \\
\hline 3010 & 9.548 & $1.06 \times 10^{-2}$ \\
\hline 1022 & 9.551 & $1.53 \times 10^{-3}$ \\
\hline 1031 & 9.580 & $2.87 \times 10^{-3}$ \\
\hline 4000 & 9.586 & $2.44 \times 10^{-3}$ \\
\hline 2012 & 9.589 & $1.76 \times 10^{-3}$ \\
\hline 1040 & 9.606 & $2.11 \times 10^{-3}$ \\
\hline 2021 & 9.618 & $4.85 \times 10^{-3}$ \\
\hline 2030 & 9.647 & $4.80 \times 10^{-3}$ \\
\hline 3011 & 9.656 & $3.51 \times 10^{-3}$ \\
\hline 3020 & 9.686 & $5.27 \times 10^{-3}$ \\
\hline 4010 & 9.724 & $2.68 \times 10^{-3}$ \\
\hline 2031 & 9.756 & $1.50 \times 10^{-3}$ \\
\hline 2040 & 9.785 & $1.15 \times 10^{-3}$ \\
\hline 3021 & 9.794 & $1.69 \times 10^{-3}$ \\
\hline 3030 & 9.823 & $1.76 \times 10^{-3}$ \\
\hline 4020 & 9.861 & $1.47 \times 10^{-3}$ \\
\hline
\end{tabular}

Our theoretical FCF calculations support all the assignments of Derrick et al. However, they also allow us to assign the experimental peak at $9.099 \mathrm{eV}$ as (0002) and the peak at $9.268 \mathrm{eV}$ as a combination of (0021) and (1002). These two very weak peaks were observed but not assigned by Derrick et al. In addition, the simulations also allow us to identify other peaks that cannot be observed separately because they are overlapped by more intense ones. For example, this occurs in the case of the (0012) peak which is overlapped by the ten times more intense (2000) peak.
We can also make predictions regarding the high energy peaks not recorded by Derrick et al. Indeed, Figs. 3 and 4, as well as the data of Table $\mathrm{V}$, show that the intensity of at least a dozen of the high energy peaks (intensity $>3.4$ ) could easily be recorded. The intensity of these high energy peaks is higher than might be expected because they are generated by the overlapping of two or more transitions. For instance, the PT2 peak predicted at $9.478 \mathrm{eV}$ is generated by the overlapping of the (1030) and (2011) transitions, with associated FCFs of $9.09 \times 10^{-3}$ and $1.12 \times 10^{-2}$, respectively.

The simulations of Trofimov et al. ${ }^{51}$ based on Poisson distributions, reproduce some features of the present simulations. Their harmonic transition frequencies agree less favorably with experiment than our PT2 values as might be expected. Trofimov et al. used the normal modes of the neutral molecule instead of the normal modes of the cation for the vibrational basis. This could be the origin of the discrepancy in their assignments of the most intense peaks as compared to us or experiment. Another difference between Trofimov et $a l$. and the present work is the very weak peak recorded at $9.099 \mathrm{eV}$ which they attribute to a vibronic interaction with the ${ }^{2} B_{1}$ electronic state whereas we have assigned this peak as (0002). Neither Trofimov et al. nor we are able to reproduce the peak located at $8.945 \mathrm{eV}$. Admittedly, however, we have not considered vibronically induced transitions.

There have also been previous theoretical simulations by Takeshita and Yamamoto ${ }^{52-54}$ that reproduced the main features of the first band in the photoelectron spectra of furan. However, the quantitative agreement with experiment is not too good due to the lack of the electron correlation effects and anharmonicity in the potential energy surface.

In order to illustrate the important role of the IFCA procedure we compare in Fig. 5 the spectra obtained using the $\mathrm{B} 3 \mathrm{LYP} / 6-311++\mathrm{G}(d, p)$ geometry for the cation with the spectra given by the IFCA procedure where the displacements of modes 4,6 , and 8 are optimized by comparison with experiment. Both simulations were done at the PT2 level using the experimental geometry for neutral furan. Figure 5 shows that using the $a b$ initio geometry for the cation leads to relative intensities that are much too weak with respect to (0000). This problem becomes more evident at higher frequency. Table VI gives IFCA values for the main geometric parameters of $\mathrm{C}_{4} \mathrm{H}_{4} \mathrm{O}^{+}$obtained by computing FCFs at the harmonic, PT1, and PT2 levels. The IFCA results can be considered valid only if they are not very different from the $a b$ initio equilibrium parameters. ${ }^{41-44}$ This is the case here as can readily be seen by comparing Tables I and VI.

Figures 6 and 7 contrast the harmonic (Fig. 6) and PT1 (Fig. 7) simulated first band of the $\mathrm{C}_{4} \mathrm{H}_{4} \mathrm{O}$ PE spectrum with the PT2 simulation, while in Tables VII and VIII we give the theoretical frequencies, relative intensities, and assignments of the simulated harmonic (PT1) spectra. When anharmonicity is omitted the relative intensities of the high frequency peaks suffer. The largest absolute difference between the harmonic and PT2 relative intensities is $8.9 \%$ for the (1020) peak which has a PT2 relative intensity of $21.2 \%$. However, the relative differences are largest for the highest frequency peaks since they have small intensity. The anharmonicity of 
TABLE IV. PT2 and experimental frequencies, relative intensities, and assignments of the low energy peaks of the $\tilde{X}^{2} A_{2} \leftarrow \tilde{X}^{1} A_{1}$ band of the furan PE spectrum.

\begin{tabular}{|c|c|c|c|c|c|}
\hline \multicolumn{3}{|c|}{ PT2 } & \multicolumn{3}{|c|}{ Expt. } \\
\hline Frequency $(\mathrm{eV})$ & Intensity & $\omega_{4} \omega_{5} \omega_{6} \omega_{8}{ }^{\mathrm{a}}$ & Frequency $(\mathrm{eV})$ & Intensity & $\omega_{4} \omega_{5} \omega_{6} \omega_{8}$ \\
\hline 8.883 & 100.0 & 0000 & 8.883 & Very strong & 0000 \\
\hline$\cdots$ & $\cdots$ & $\cdots$ & 8.945 & Very weak & $\cdots$ \\
\hline 8.991 & 26.9 & 0001 & 8.987 & Medium & 0001 \\
\hline 9.020 & 60.5 & 0010 & 9.016 & Strong & 0010 \\
\hline 9.059 & 73.7 & 1000 & 9.059 & Strong & 1000 \\
\hline 9.100 & 3.7 & 0002 & 9.099 & Very weak & $\cdots$ \\
\hline 9.128 & 17.4 & 0011 & 9.124 & Weak & 0011 \\
\hline 9.162 & 29.6 & 0020,1001 & 9.155 & Medium & 0020,1001 \\
\hline 9.196 & 48.3 & 1010 & 9.193 & Medium & 1010 \\
\hline 9.235 & 29.8 & 2000, 0012 & 9.233 & Medium & 2000 \\
\hline 9.268 & 7.3 & 0021, 1002 & 9.271 & Very weak & $\cdots$ \\
\hline
\end{tabular}

${ }^{\mathrm{a}}$ The highest FCF associated with each peak is indicated with a bold label. Only the FCF large than $10^{-3}$ units has been used to label the peaks.

the PES also decreases the frequency of the peaks, with a larger reduction for the high energy peaks. For instance, the decreases for the low, medium, and high energy peaks (0001), (2010), and (1031) are, respectively, 1, 7, and $15 \mathrm{meV}$.

At the PT1 level the frequencies are unaffected by anharmonicity. On the other hand, the relative intensities incorporate about $70 \%$ of the anharmonicity effect. Whereas the sum of the absolute differences between the harmonic and PT2 relative intensities of the first 26 peaks is 75.1 [as compared to 100.0 for the intensity of the (0000) peak], the sum of the absolute differences between PT1 and PT2 is only 21.8. The largest absolute difference between PT1 and PT2 is 3.7 for the (1001) peak. The good performance of PT1 in calculating FCFs agrees with our previous results for $\mathrm{ClO}_{2}$ (Ref. 20) and $\mathrm{C}_{2} \mathrm{H}_{4} \cdot{ }^{17}$

TABLE V. PT2 frequencies, relative intensities, and assignments of the high energy peaks of the $\tilde{X}^{2} A_{2} \leftarrow \tilde{X}^{1} A_{1}$ band of the furan PE spectrum. Only the peaks with a relative intensity larger than $1 \%$ of the (0000) peak are shown.

\begin{tabular}{ccc}
\hline \hline Frequency $(\mathrm{eV})$ & Intensity & $\omega_{4} \omega_{5} \omega_{6} \omega_{8}{ }^{\mathrm{a}}$ \\
\hline 9.034 & 16.1 & $0030, \mathbf{1 0 1 1}$ \\
9.336 & 21.2 & $\mathbf{1 0 2 0}, 2001$ \\
9.372 & 21.0 & $\mathbf{2 0 1 0}, 0022$ \\
9.411 & 9.7 & $0031, \mathbf{3 0 0 0}, 1012$ \\
9.442 & 6.5 & $0040, \mathbf{1 0 2 1}, 2002$ \\
9.478 & 8.9 & $1030, \mathbf{2 0 1 1}$ \\
9.511 & 10.0 & $\mathbf{2 0 2 0}, 3001$ \\
9.548 & 7.1 & $\mathbf{3 0 1 0}, 1022$ \\
9.584 & 3.4 & $\mathbf{1 0 3 1}, 4000,2012$ \\
9.617 & 3.4 & $1040, \mathbf{2 0 2 1}$ \\
9.650 & 3.9 & $\mathbf{2 0 3 0}, 3011$ \\
9.686 & 3.6 & $\mathbf{3 0 2 0}$ \\
9.724 & 2.2 & $\mathbf{4 0 1 0}$ \\
9.758 & 1.2 & $\mathbf{2 0 3 1}$ \\
9.792 & 1.3 & $2040, \mathbf{3 0 2 1}$ \\
9.826 & 1.2 & $\mathbf{3 0 3 0}$ \\
9.861 & 1.0 & $\mathbf{4 0 2 0}$ \\
\hline \hline
\end{tabular}

${ }^{\mathrm{a}}$ The highest FCF associated with each peak is indicated with a bold label Only the FCF larger than $10^{-3}$ units has been used to label the peaks.
Finally, we explore the influence of deuteration. Even though the low experimental resolution realized by Rennie et $a .^{55,56}$ (cf. Fig. 8) makes assignments more difficult, they were able to identify two progressions involving just two $a_{1}$ normal modes. Both progressions involve the excitation of mode 4 with a fundamental frequency of $911 \mathrm{~cm}^{-1}$. The most intense has no other modes excited whereas the second progression involves a single excitation of mode 6 with a fundamental frequency of $1354 \mathrm{~cm}^{-1}$ [i.e., $\left.\left(\omega_{4}, 1\right)\right]$.

In the present study we simulated the first band of the photoelectron spectrum of deuterated furan at the harmonic level. Since the resolution is relatively poor we did not bother to reoptimize the geometry of the cation using the IFCA procedure. That is to say, we employed the IFCA geometry obtained in our $\mathrm{C}_{4} \mathrm{H}_{4} \mathrm{O}$ calculations. This approximation, together with the harmonic approximation, does not allow us to reproduce peak intensities quantitatively, but it is accurate enough to check the experimental assignments and to see the major effects of deuteration.

Our calculations reveal that, in fact, four modes are responsible for the intensities in the photoelectron spectra of $\mathrm{C}_{4} \mathrm{D}_{4} \mathrm{O}$. In the last two columns of Table II we present our $a b$ initio harmonic frequencies for neutral and cationic deuterated furan. The four $a_{1}$ normal modes of the cation that generate non-negligible FCF in the simulated first band of the photoelectron spectrum (see Fig. 9) are modes 3, 4, 5, and 6 with calculated harmonic frequencies of 1437, 1366, 1102, and $922 \mathrm{~cm}^{-1}$, respectively. A comparison of Figs. 2 and 9 shows that the active modes in deuterated furan are quite dissimilar from the active modes in the normal species.

In Table IX we present our assignments for the first 16 peaks of the spectrum and in Fig. 10 we compare our simulation of the $\mathrm{C}_{4} \mathrm{D}_{4} \mathrm{O}$ band with that of $\mathrm{C}_{4} \mathrm{H}_{4} \mathrm{O}$. The theoretical data show that the most intense progression of peaks is given by simultaneous excitation of $\omega_{3}$ and $\omega_{4}$ [i.e., $\left.\left(\omega_{3} \omega_{4} 00\right)\right]$. Thus, the first peak of this progression is given by transitions where $\omega_{3}+\omega_{4}=1$ [i.e., (0100) and (1000)], the second peak by transitions where $\omega_{3}+\omega_{4}=2$ [i.e., (0200), (1100), and (2000)], and so on. The intensity of transitions where $\omega_{4}$ is excited is slightly greater than the corresponding 


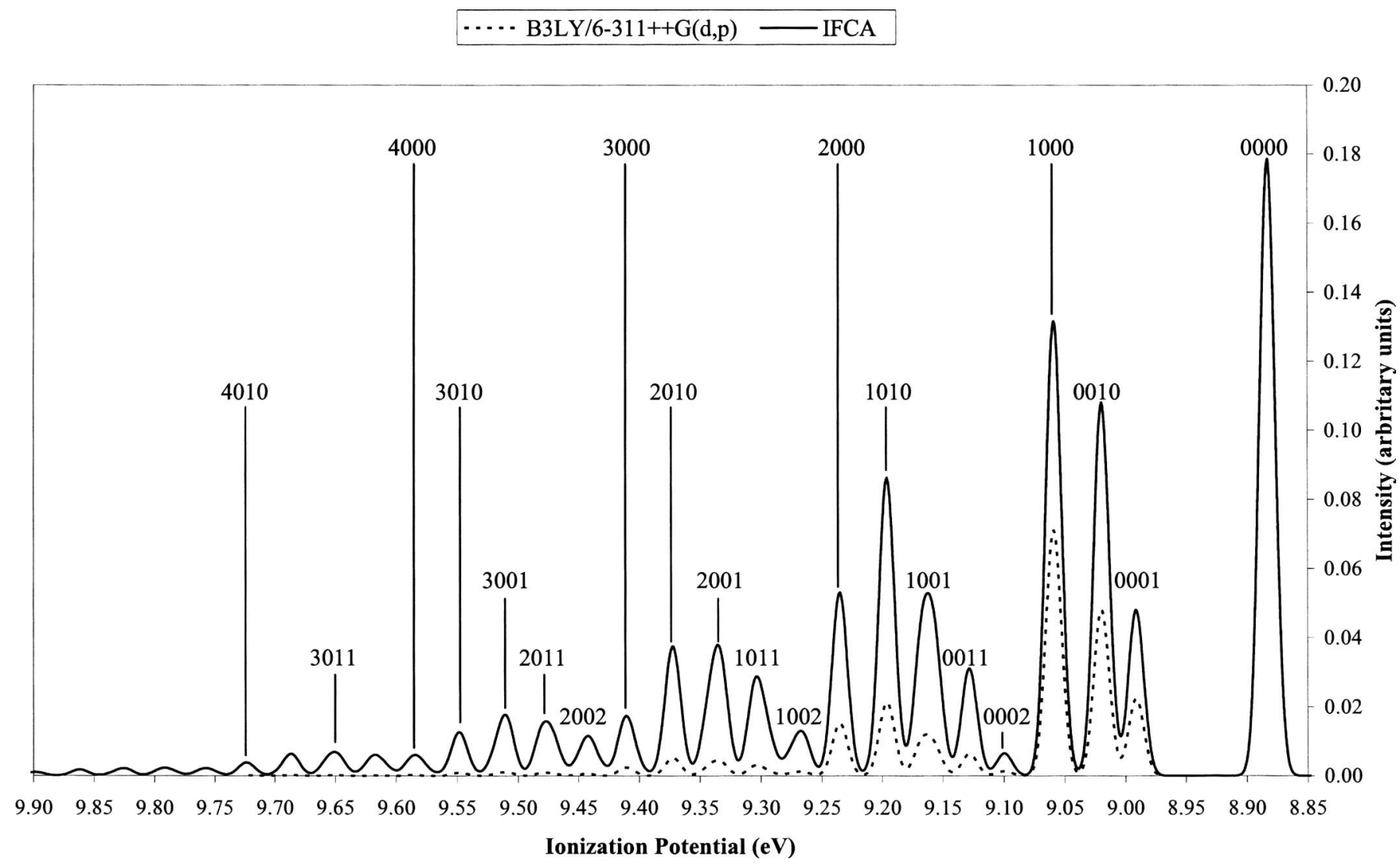

FIG. 5. PT2 simulation of the first band in the furan PE spectrum. The dashed and solid lines represent the spectra obtained using the (U)B3LYP/6-311+ $+\mathrm{G}(d, p)$ equilibrium cation geometry and IFCA cation geometry, respectively.

transitions where $\omega_{3}$ is excited. The second progression is given by $\left(\omega_{3} \omega_{4} 01\right)$ transitions starting with $(0001)$. A third low intensity progression, not identified by Rennie et al., is $\left(\omega_{3} \omega_{4} 02\right)$. There are still other progressions with lower intensity that are overlapped by these three. For example, $\left(\omega_{3} \omega_{4} 03\right)$ is overlapped by $\left(\omega_{3} \omega_{4} 00\right)$. Finally, the mode $\omega_{5}$ generates only very low intensity FCFs. Nonetheless, the (0010) and (0011) peaks can readily be identified in the theoretical spectra.

Our theoretical simulation for the first band of the $\mathrm{C}_{4} \mathrm{D}_{4} \mathrm{O}$ photoelectron spectrum reflects all the important changes from $\mathrm{C}_{4} \mathrm{H}_{4} \mathrm{O}$ observed by Rennie et al. For instance, in $\mathrm{C}_{4} \mathrm{H}_{4} \mathrm{O}$ the (0010) peak is the third most intense whereas in $\mathrm{C}_{4} \mathrm{D}_{4} \mathrm{O}$ this peak is so weak that it was not recorded in the

TABLE VI. IFCA geometrical parameters for cationic furan. All quantities are in angstroms and degrees (see Fig. 1).

\begin{tabular}{cccc}
\hline \hline & Harmonic & PT1 & PT2 \\
\hline$r(1 \mathrm{O}-2 \mathrm{C})$ & 1.342 & 1.340 & 1.339 \\
$r(2 \mathrm{C}-3 \mathrm{C})$ & 1.431 & 1.436 & 1.437 \\
$r(3 \mathrm{C}-4 \mathrm{C})$ & 1.372 & 1.369 & 1.368 \\
$r(2 \mathrm{C}-6 \mathrm{H})$ & 1.082 & 1.083 & 1.083 \\
$r(3 \mathrm{C}-7 \mathrm{H})$ & 1.081 & 1.081 & 1.081 \\
$\angle(5 \mathrm{C}-1 \mathrm{O}-2 \mathrm{C})$ & 106.7 & 106.7 & 106.8 \\
$\angle(1 \mathrm{O}-2 \mathrm{C}-3 \mathrm{C})$ & 110.9 & 110.9 & 110.8 \\
$\angle(2 \mathrm{C}-3 \mathrm{C}-4 \mathrm{C})$ & 105.8 & 105.8 & 105.8 \\
$\angle(1 \mathrm{O}-2 \mathrm{C}-6 \mathrm{H})$ & 116.6 & 116.7 & 116.9 \\
$\angle(2 \mathrm{C}-3 \mathrm{C}-7 \mathrm{H})$ & 125.5 & 125.5 & 125.4 \\
\hline \hline
\end{tabular}

experimental spectrum. In contrast with the analysis of Rennie et al., however, the theoretical simulation indicates clearly that not two, but four, modes are active in the $\tilde{X}^{2} A_{2} \leftarrow \tilde{X}^{1} A_{1}$ band of deuterated furan.

TABLE VII. Harmonic frequencies, relative intensities, and assignments of the peaks with a relative intensity larger than $1 \%$ of the (0000) peak in the $\tilde{X}^{2} A_{2} \leftarrow \widetilde{X}^{1} A_{1}$ band of the furan PE spectrum.

\begin{tabular}{ccc}
\hline \hline Frequency $(\mathrm{eV})$ & Intensity & $\omega_{4} \omega_{5} \omega_{6} \omega_{8}{ }^{\mathrm{a}}$ \\
\hline 8.883 & 100.0 & $\mathbf{0 0 0 0}$ \\
8.992 & 26.9 & $\mathbf{0 0 0 1}$ \\
9.021 & 60.5 & $\mathbf{0 0 1 0}$ \\
9.062 & 73.7 & $\mathbf{1 0 0 0}$ \\
9.102 & 3.5 & $\mathbf{0 0 0 2}$ \\
9.131 & 15.5 & $\mathbf{0 0 1 1}$ \\
9.168 & 23.1 & $0020, \mathbf{1 0 0 1}$ \\
9.200 & 41.7 & $\mathbf{1 0 1 0}$ \\
9.241 & 29.0 & $0012, \mathbf{2 0 0 0}$ \\
9.271 & 4.6 & $\mathbf{0 0 2 1}, 1002$ \\
9.309 & 10.8 & $0030, \mathbf{1 0 1 1}$ \\
9.340 & 12.3 & $\mathbf{1 0 2 0}, 2001$ \\
9.379 & 14.8 & $0022, \mathbf{2 0 1 0}$ \\
9.420 & 7.9 & $0031,1012, \mathbf{3 0 0 0}$ \\
9.448 & 2.8 & $\mathbf{1 0 2 1}, 2002$ \\
9.487 & 3.8 & $1030, \mathbf{2 0 1 1}$ \\
9.519 & 3.9 & $\mathbf{2 0 2 0}, 3001$ \\
9.558 & 3.6 & $\mathbf{3 0 1 0}$ \\
9.599 & 1.7 & $\mathbf{4 0 0 0}$ \\
\hline
\end{tabular}

$\overline{\bar{a} \text { The highest FCF associated with each peak is indicated with a bold label. }}$ Only the FCF larger than $10^{-3}$ units has been used to label the peaks. 


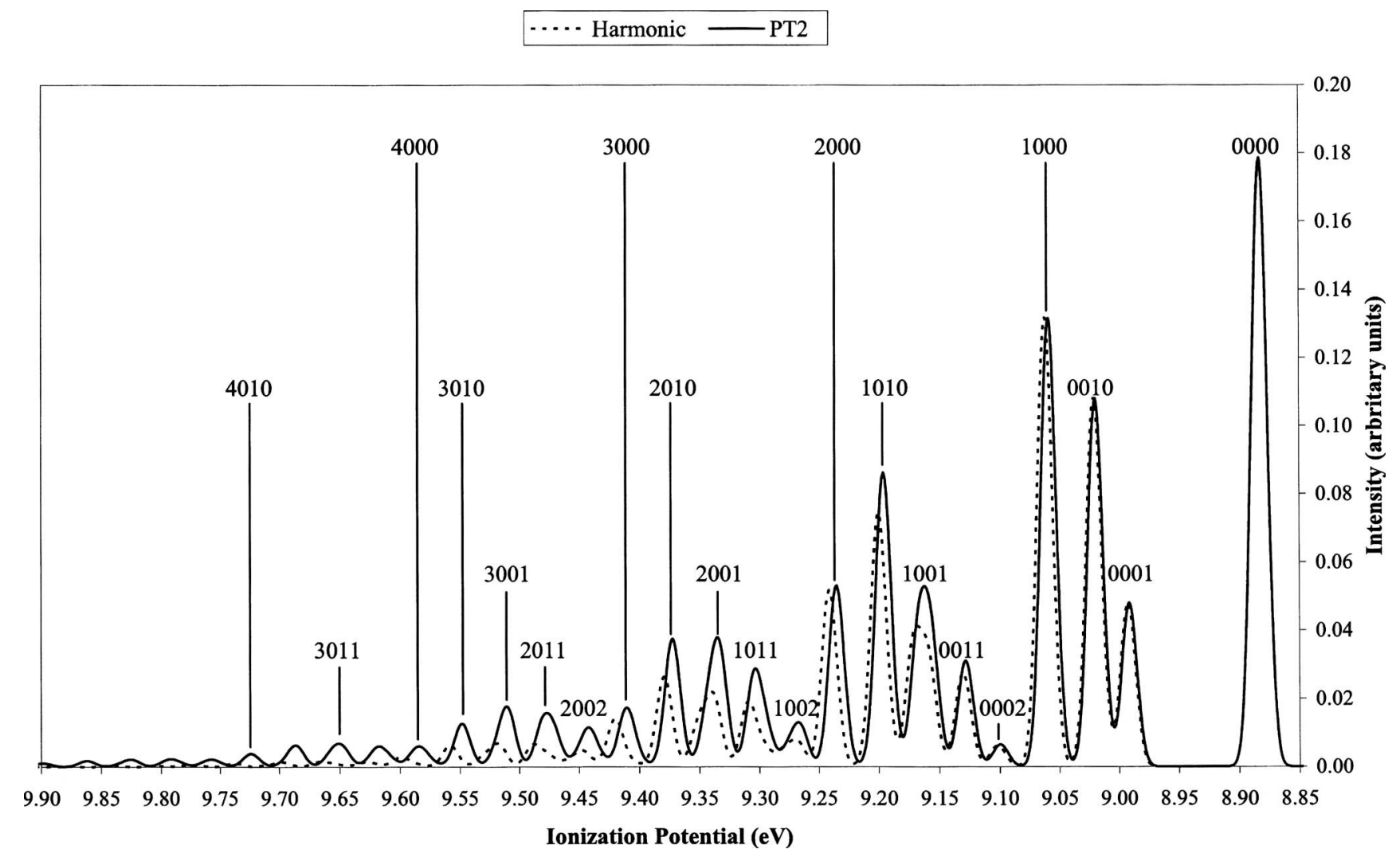

FIG. 6. (U)B3LYP/6-311++G(d,p) simulation of the first band in the furan PE spectrum. The dashed and solid lines represent the harmonic and PT2 anharmonic spectra, respectively.

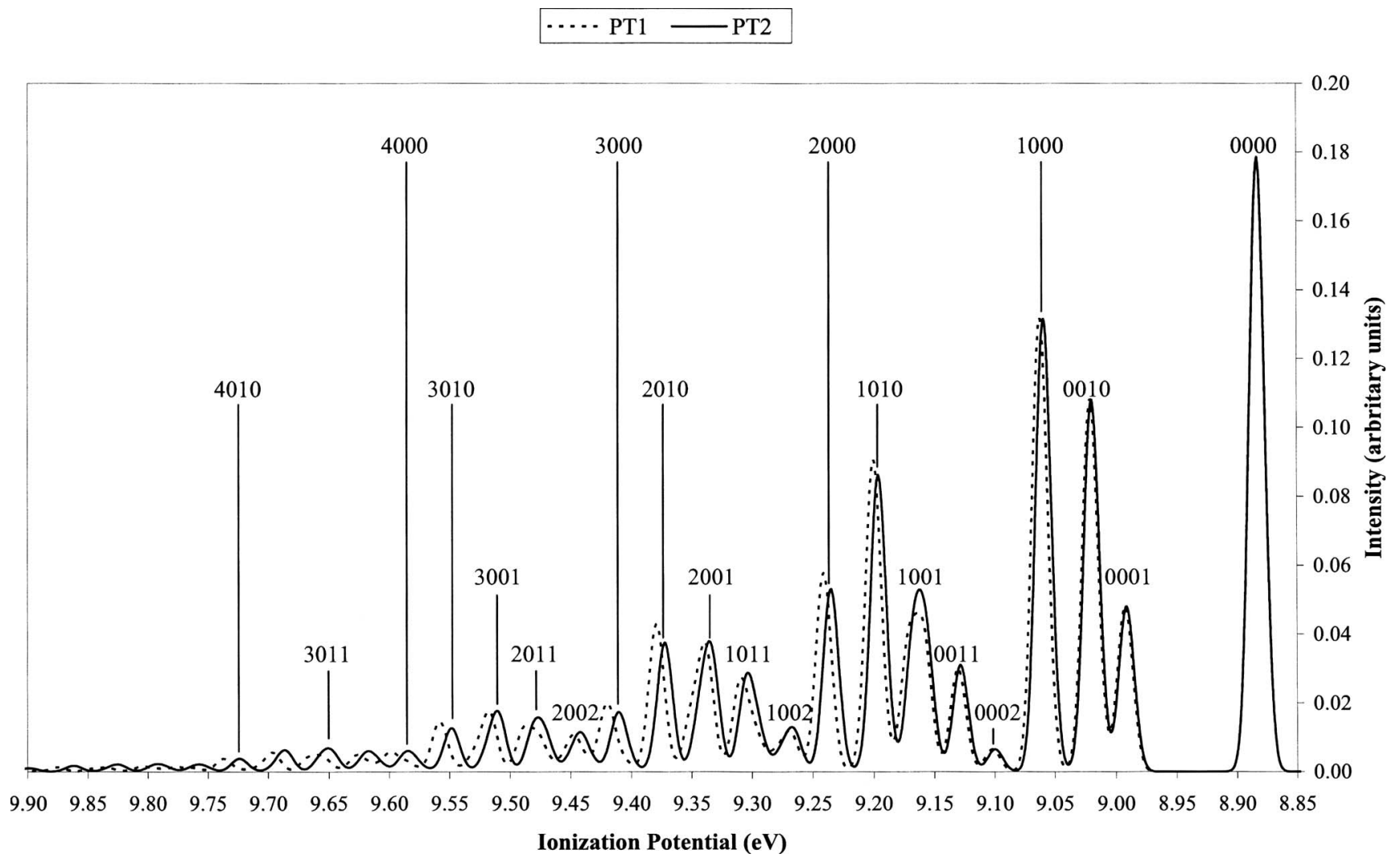

FIG. 7. (U)B3LYP/6-311++G(d,p) simulation of the first band in the furan PE spectrum. The dashed and solid lines represent the PT1 and PT2 anharmonic spectra, respectively. 
TABLE VIII. PT1 frequencies, relative intensities, and assignments of the peaks with a relative intensity larger than $1 \%$ of the $(0000)$ peak in the $\tilde{X}^{2} A_{2} \leftarrow \tilde{X}^{1} A_{1}$ bond of the furan PE spectrum.

\begin{tabular}{ccc}
\hline \hline Frequency $(\mathrm{eV})$ & Intensity & $\omega_{4} \omega_{5} \omega_{6} \omega_{8}{ }^{\mathrm{a}}$ \\
\hline 8.883 & 100.0 & $\mathbf{0 0 0 0}$ \\
8.992 & 26.9 & $\mathbf{0 0 0 1}$ \\
9.021 & 60.5 & $\mathbf{0 0 1 0}$ \\
9.062 & 73.7 & $\mathbf{1 0 0 0}$ \\
9.102 & 3.7 & $\mathbf{0 0 0 2}$ \\
9.131 & 16.9 & $\mathbf{0 0 1 1}$ \\
9.163 & 25.9 & $\mathbf{0 0 2 0}, 1001$ \\
9.200 & 50.7 & $\mathbf{1 0 1 0}$ \\
9.241 & 32.3 & $0012, \mathbf{2 0 0 0}$ \\
9.270 & 6.7 & $\mathbf{0 0 2 1}, 1002$ \\
9.308 & 15.4 & $0030, \mathbf{1 0 1 1}$ \\
9.340 & 21.1 & $\mathbf{1 0 2 0}, 2001$ \\
9.379 & 24.0 & $0022, \mathbf{2 0 1 0}$ \\
9.420 & 11.2 & $0031,1012, \mathbf{3 0 0 0}$ \\
9.448 & 6.1 & $\mathbf{1 0 2 1}, 2002,0040$ \\
9.486 & 7.7 & $1030, \mathbf{2 0 1 1}$ \\
9.517 & 9.9 & $\mathbf{2 0 2 0}, 3001$ \\
9.558 & 8.2 & $\mathbf{3 0 1 0}, 1022$ \\
9.598 & 3.3 & $1031,2012, \mathbf{4 0 0 0}$ \\
9.626 & 2.8 & $1040, \mathbf{2 0 2 1}$ \\
9.659 & 2.8 & $\mathbf{2 0 3 0}, 3011$ \\
9.697 & 3.2 & $\mathbf{3 0 2 0}, 4001$ \\
9.738 & 2.2 & $\mathbf{4 0 1 0}$ \\
\hline \hline
\end{tabular}

${ }^{\mathrm{a}}$ The highest FCF associated with each peak is indicated with a bold label. Only the FCF larger than $10^{-3}$ units has been used to label the peaks.

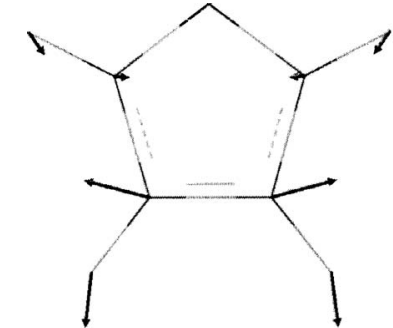

(A)

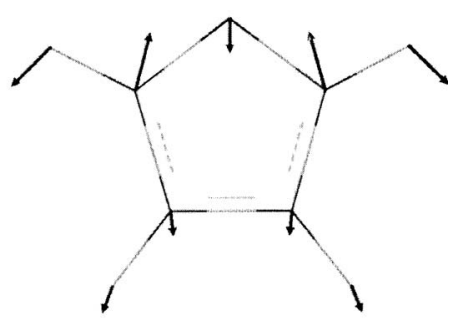

(B)

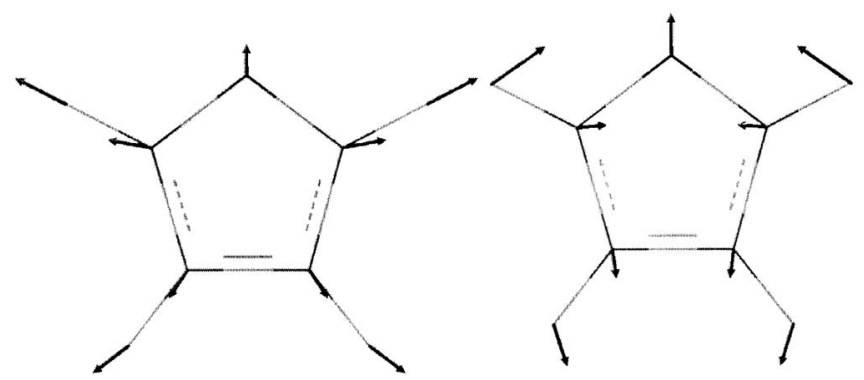

(C)

(D)

FIG. 9. Displacements associated with $a_{1} \omega_{3}(\mathrm{~A}), \omega_{4}(\mathrm{~B}), \omega_{5}(\mathrm{C})$, and $\omega_{6}(\mathrm{D})$ normal modes of $\mathrm{C}_{4} \mathrm{D}_{4} \mathrm{O}$.

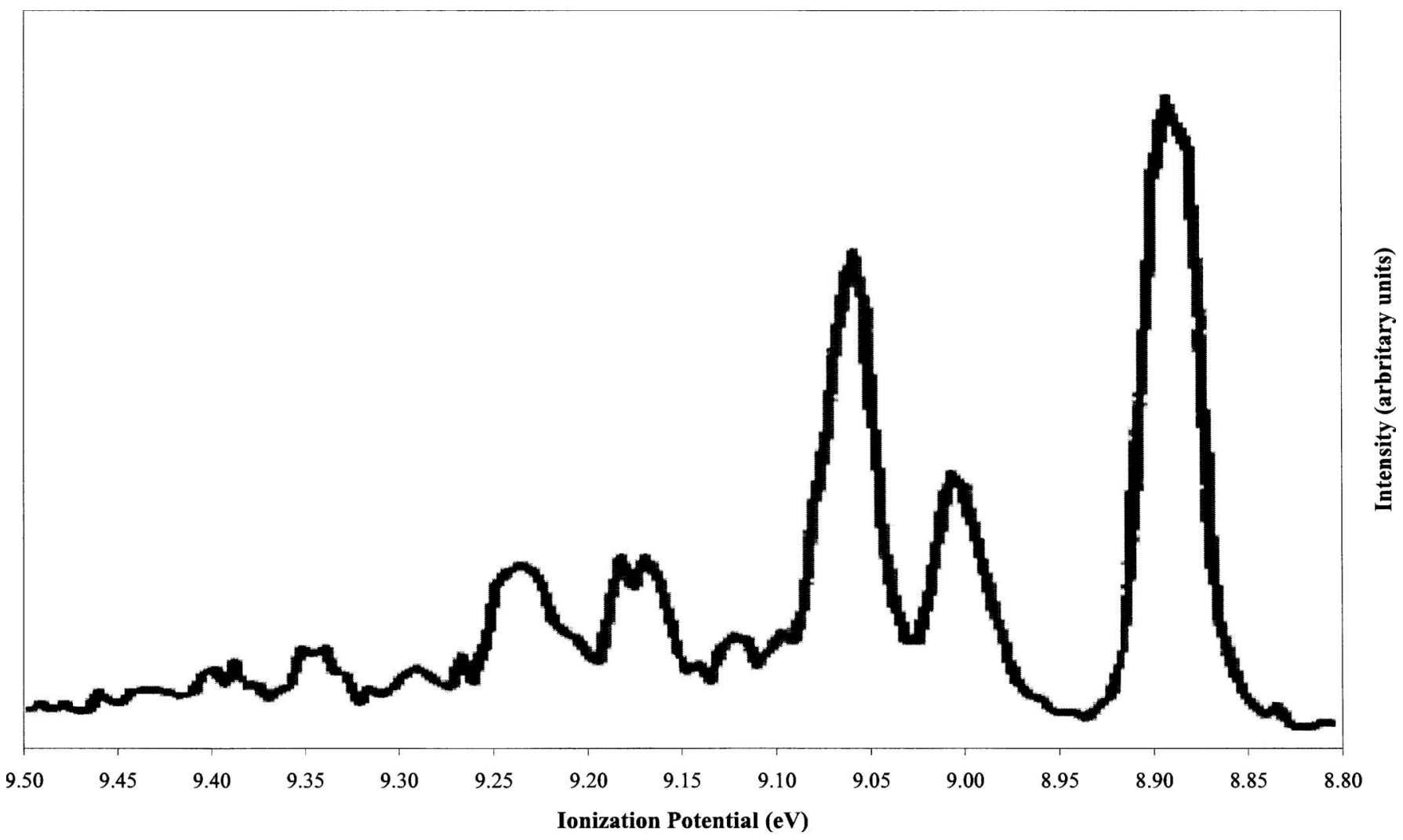

FIG. 8. Experimental first band of the deuterated furan PE spectrum measured by Rennie et al. (Refs. 55 and 56). 
TABLE IX. Harmonic frequencies, relative intensities, and assignments of the peaks with a relative intensity larger than $1 \%$ of the $(0000)$ peak in the $\tilde{X}^{2} A_{2} \leftarrow \tilde{X}^{1} A_{1}$ band of the deuterated furan PE spectrum.

\begin{tabular}{|c|c|c|}
\hline Frequency $(\mathrm{eV})$ & Intensity & $\omega_{3} \omega_{4} \omega_{5} \omega_{6}^{\mathrm{a}}$ \\
\hline 8.883 & 100.0 & 0000 \\
\hline 8.997 & 56.3 & 0001 \\
\hline 9.019 & 5.6 & 0010 \\
\hline 9.056 & 86.3 & 0100, 1000 \\
\hline 9.112 & 15.4 & 0002 \\
\hline 9.134 & 2.6 & 0011 \\
\hline 9.170 & 47.1 & $\mathbf{0 1 0 1}, 1001,0110,1010$ \\
\hline 9.229 & 43.0 & $\begin{array}{c}0200,0003, \mathbf{1 1 0 0}, 2000 \\
0012\end{array}$ \\
\hline 9.285 & 12.4 & $\mathbf{0 1 0 2}, 1002,0111,1011$ \\
\hline 9.344 & 21.9 & $0201, \mathbf{1 1 0 1}, 2001,1110$ \\
\hline 9.403 & 15.2 & $\begin{array}{c}0300,0103, \mathbf{1 2 0 0}, 1003, \\
2100,3000\end{array}$ \\
\hline 9.459 & 5.5 & 0202, 1102, 2002 \\
\hline 9.519 & 7.1 & $0301,1201, \mathbf{2 1 0 1}, 3001$ \\
\hline 9.577 & 4.2 & $\begin{array}{c}1300,1103, \mathbf{2 2 0 0}, 3100 \\
4000\end{array}$ \\
\hline 9.634 & 1.7 & 1202, 2102 \\
\hline 9.693 & 1.8 & 1301, 2201, 3101 \\
\hline
\end{tabular}

$\overline{\bar{a} \text { The highest FCF associated with each peak is indicated with a bold label. }}$ Only the FCF larger than $10^{-3}$ units has been used to label the peaks.

\section{CONCLUSIONS}

We have applied our new procedure for calculating Franck-Condon factors to simulate the $\mathrm{C}_{4} \mathrm{H}_{4} \mathrm{O}^{+} \widetilde{X}^{2} A_{2}$ $\leftarrow \mathrm{C}_{4} \mathrm{H}_{4} \mathrm{O} \tilde{X}^{1} A_{1}$ band in the photoelectron spectrum. This simulation takes into account anharmonic mode-mode coupling as well as Duschinsky rotations at the second-order perturbation theory level. At the present time there is no other practical procedure for incorporating these effects for larger than tetraatomic systems.

A key feature of the treatment is an efficient algorithm for generating the minimal sufficient vibrational basis set. In the case of furan this algorithm leads to a basis set containing excitations of just three $a_{1}$ modes. A fourth mode is needed to obtain the anharmonic frequencies but not the FranckCondon factors themselves. Using these four modes and the IFCA geometry of the cation the observed spectrum is reproduced quite accurately. As a result we were able to improve upon and extend previous assignments as well as suggest further measurements. It turns out that most of the effect of anharmonicity in the FCFs is already included at the PT1 level. However, PT2 is needed for the vibrational frequencies since the PT1 correction vanishes.

Although the IFCA is essential for quantitative results, as we have shown, it is feasible for interpretive purposes to

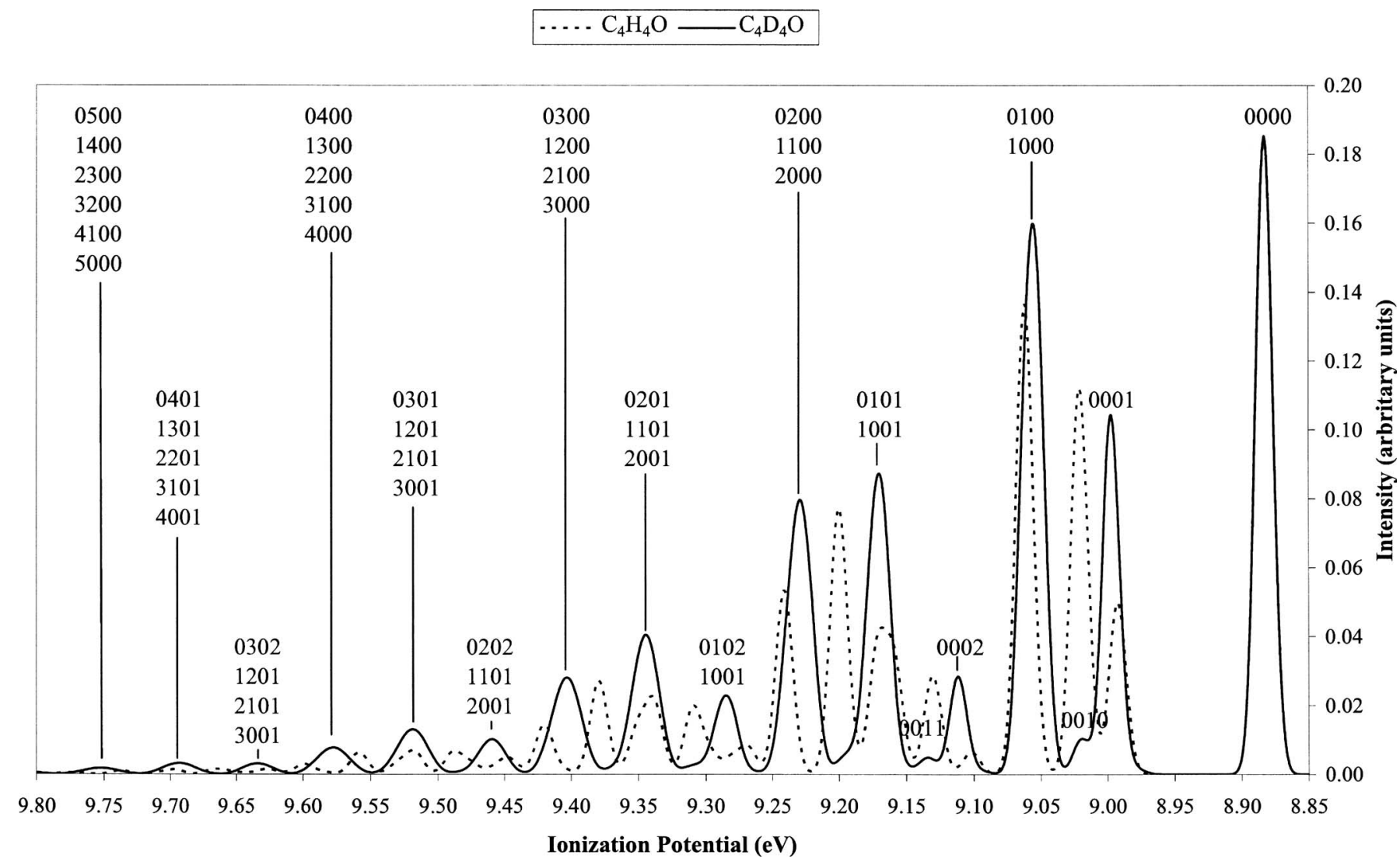

FIG. 10. Comparison of the PT2 simulation for the first band in the $\mathrm{C}_{4} \mathrm{H}_{4} \mathrm{O}$ PE spectrum (dashed line) with the corresponding band of the $\mathrm{C}_{4} \mathrm{D}_{4} \mathrm{O} P E$ spectrum (solid line). 
do without applying that procedure. This was demonstrated in the case of deuterated furan where we were able to correct previous misassignments based on low resolution photoelectron spectra.

It is possible that second- (or higher-) order perturbation theory may not behave as well for other systems as it does for furan. Slow convergence or divergence could occur for systems with large anharmonicity and/or large numbers of strongly coupled vibrations. In order to overcome this potential problem we are currently working on a variational approach to replace our perturbation treatment.

\section{ACKNOWLEDGMENTS}

Support for this work under The Spanish Ministerio de Educación y Ciencia (MEC) Project No. CTQ2005-08797C02-01/BQU and by the DURSI Project No. 2005SGR00238 is acknowledged. Three of the authors (N.S.B., B.K., and J.M.L.) thank financial support from the Generalitat de Catalunya through the Gaspar de Portolà programs.

${ }^{1}$ J. Franck, Trans. Faraday Soc. 21, 536 (1925).

${ }^{2}$ E. U. Condon, Phys. Rev. 32, 858 (1928).

${ }^{3}$ G. Herzberg and E. Teller, Z. Phys. Chem. Abt. B 21, 410 (1933).

${ }^{4}$ H. Köppel, W. Domcke, and L. S. Cederbaum, Adv. Chem. Phys. 57, 59 (1984)

${ }^{5}$ T. E. Sharp and H. M. Rosenstock, J. Chem. Phys. 41, 3453 (1964).

${ }^{6}$ E. V. Doctorov, I. A. Malkin, and V. I. Man'ko, J. Mol. Spectrosc. 64, 302 (1977).

${ }^{7}$ P. Chen, in Unimolecular and Bimolecular Ion-Molecule Reaction Dynamics, edited by C.-Y. Ng, T. Baer, and I. Powis (Wiley, Chichester, 1994), p. 371.

${ }^{8}$ M. Dierksen and S. Grimme, J. Chem. Phys. 122, 244101 (2005).

${ }^{9}$ F. Iachello and S. Oss, Phys. Rev. Lett. 66, 2976 (1991).

${ }^{10}$ B. Segev and E. J. Heller, J. Chem. Phys. 112, 4004 (2000).

${ }^{11}$ S. Kallush, B. Segev, A. V. Sergeev, and E. J. Heller, J. Phys. Chem. A 106, 6006 (2002)

${ }^{12}$ K. M. Ervin, T. M. Ramond, G. E. Davico, R. L. Schwartz, S. M. Casey, and W. C. Lineberger, J. Phys. Chem. A 105, 10822 (2001).

${ }^{13}$ H. Kikuchi, M. Kubo, N. Watanabe, and H. Suzuki, J. Chem. Phys. 119, 729 (2003).

${ }^{14}$ A. Hazra and M. Nooijen, J. Chem. Phys. 122, 204327 (2005).

${ }^{15}$ J. Neugebauera, E. J. Baerends, M. Nooijen, and J. Autschbach, J. Chem. Phys. 122, 234305 (2005).

${ }^{16}$ K. Umesaki and H. Kikuchi, J. Chem. Phys. 124, 074304 (2006).

${ }^{17}$ J. M. Luis, M. Torrent-Sucarrat, M. Solà, D. M. Bishop, and B. Kirtman, J. Chem. Phys. 122, 184104 (2005).

${ }^{18}$ J. M. Bowman, X. C. Huang, L. B. Harding, and S. Carter, Mol. Phys. 104, 33 (2006).

${ }^{19}$ F. T. Chau, D. K. W. Mok, E. P. F. Lee, and J. M. Dyke, ChemPhysChem 6, 2037 (2005)

${ }^{20}$ J. M. Luis, D. M. Bishop, and B. Kirtman, J. Chem. Phys. 120, 813 (2004).
${ }^{21}$ J. M. Bowman, J. Chem. Phys. 68, 608 (1978).

${ }^{22}$ R. B. Gerber and M. A. Ratner, Chem. Phys. Lett. 68, 195 (1979).

${ }^{23}$ J. M. Bowman, Acc. Chem. Res. 19, 202 (1986).

${ }^{24}$ R. B. Gerber and M. A. Ratner, Adv. Chem. Phys. 70, 97 (1988)

${ }^{25}$ J.-Q. Jung and R. B. Gerber, J. Chem. Phys. 105, 10332 (1996).

${ }^{26}$ G. M. Gaban, J. Phys. Chem. A 108, 4551 (2004).

${ }^{27}$ J. M. Bowman, K. M. Christoffel, and F. Tobin, J. Phys. Chem. 83, 905 (1979).

${ }^{28}$ K. M. Christoffel and J. M. Bowman, Chem. Phys. Lett. 85, 220 (1982).

${ }^{29}$ S. Carter, J. M. Bowman, and N. C. Handy, Theor. Chim. Acta 100, 191 (1998).

${ }^{30}$ K. Yagi, T. Taketsugu, K. Hirao, and M. S. Gordon, J. Chem. Phys. 113, 1005 (2000).

${ }^{31}$ V. Barone, J. Chem. Phys. 120, 3059 (2004).

${ }^{32}$ O. Christiansen, J. Chem. Phys. 120, 2149 (2004).

${ }^{33}$ A. D. Becke, J. Chem. Phys. 98, 5648 (1993).

${ }^{34}$ C. Lee, W. Yang, and R. G. Parr, Phys. Rev. B 37, 785 (1988).

${ }^{35}$ R. Krishnan, J. S. Binkley, R. Seeger, and J. A. Pople, J. Chem. Phys. 72, 650 (1980).

${ }^{36}$ T. Clark, J. Chandrasekhar, and P. v. R. Schleyer, J. Comput. Chem. 4, 294 (1983).

${ }^{37}$ M. J. Frisch, G. W. Trucks, H. B. Schlegel et al., GAUSSIAN 03, Revision B.02, Gaussian, Inc., Pittsburgh, PA, 2003.

${ }^{38}$ P. J. Davis and P. Rabinowitz, Numerical Integration (Blaisdell, London, 1967), p. 166

${ }^{39}$ P. J. Derrick, L. Åsbrink, O. Edqvist, B.-Ö. Jonsson, and E. Lindholm, Int. J. Mass Spectrom. Ion Phys. 6, 161 (1971).

${ }^{40}$ F. Mata, M. C. Martin, and G. O. Sörensen, J. Mol. Struct. 48, 157 (1978).

${ }^{41}$ E. P. F. Lee, D. K. W. Mok, F.-T. Chau, and J. M. Dyke, J. Chem. Phys. 121, 2962 (2004)

${ }^{42}$ F.-T. Chau, D. K. W. Mok, E. P. F. Lee, and J. M. Dyke, J. Chem. Phys. 121, 1810 (2004).

${ }^{43}$ N. Bulcourt, J.-P. Booth, E. A. Hudson, J. Luque, D. K. W. Mok, E. P. Lee, F.-T. Chau, and J. M. Dyke, J. Chem. Phys. 120, 9499 (2004).

${ }^{44}$ D. K. W. Mok, E. P. F. Lee, F.-T. Chau, and J. M. Dyke, J. Chem. Phys. 120, 1292 (2004)

${ }^{45}$ S. Yan, Y. Bu, and L. Sun, J. Mol. Struct. 671, 161 (2004).

${ }^{46}$ R. Burcl, R. D. Amos, and N. C. Handy, Chem. Phys. Lett. 355, 8 (2002).

${ }^{47}$ A. Mellouki, J. Liévin, and M. Herman, Chem. Phys. 271, 239 (2001).

${ }^{48}$ E. V. Gromov, A. B. Trofimov, N. M. Vitkovskaya, J. Schirmer, and H. Köppel, J. Chem. Phys. 119, 737 (2003).

${ }^{49}$ L. Nygaard, J. T. Nielsen, J. Kirchheimer, G. Maltesen, N. RastrupAndersen, and G. O. Sorensen, J. Mol. Struct. 3, 491 (1969).

${ }^{50}$ A. A. El-Azhary and H. U. Suter, J. Phys. Chem. 100, 15056 (1996)

${ }^{51}$ A. B. Trofimov, H. Köppel, and J. Schirmer, J. Chem. Phys. 109, 1025 (1998).

${ }^{52}$ K. Takeshita and Y. Yamamoto, Theor. Chim. Acta 92, 199 (1995).

${ }^{53}$ K. Takeshita and Y. Yamamoto, J. Chem. Phys. 101, 2198 (1994).

${ }^{54}$ K. Takeshita and Y. Yamamoto, Chem. Phys. 189, 489 (1994).

${ }^{55}$ E. E. Rennie, C. A. F. Johnson, J. E. Parker, D. M. P. Holland, D. A. Shaw, M. A. MacDonald, M. A. Hayes, and L. G. Shpinkova, Chem. Phys. 236, 365 (1998).

${ }^{56}$ E. E. Rennie, L. Cooper, C. A. F. Johnson, J. E. Parker, R. A. Mackie, L. G. Shpinkova, D. M. P. Holland, D. A. Shaw, and M. A. Hayes, Chem. Phys. 263, 149 (2001). 